\title{
Long-Term Neurophysiological Outcomes in Patients Undergoing Coronary Artery Bypass Grafting
}

\author{
Irina V. Tarasova ${ }^{1}$, MD, PhD; Olga A. Trubnikova ${ }^{1}$, MD, PhD; Irina D. Syrova ${ }^{1}$, MD, PhD; Olga L. Barbarash , MD, PhD
}

DOI: $10.21470 / 1678-9741-2020-0390$

\begin{abstract}
Introduction: This study aims to evaluate late postoperative neurophysiological outcomes in patients after coronary artery bypass grafting (CABG).

Methods: Forty-five male patients with stable coronary artery disease aged 45-69 years underwent extended neuropsychological assessment using the software Status PF and electroencephalographical examination 3-5 days before CABG and 5-7 years after CABG. Postoperative decline in cognitive functions was determined by a $20 \%$ decrease in the cognitive indicator compared to that at baseline on $20 \%$ of the tests included in the Status PF battery. Statistical analysis was performed using the software STATISTICA 10.0. Multiple regression was used to identify demographic, clinical, and electroencephalographical variables associated with adverse cognitive outcomes.

Results: Cognitive decline was observed in $54 \%$ of the patients in the long-term postoperative period. Five to seven
\end{abstract}

years after CABG, all patients have shown an increase in the theta rhythm power compared to the preoperative values, which is most pronounced in the frontal and temporal areas of the right hemisphere $(P=0.04)$, along with a decrease in the alpha rhythm in the posterior areas of the cortex $(P=0.005)$. Multiple regression has reported that the main predictors of cognitive impairment are slower mean alpha frequency, decreased theta- 2 rhythm with eyes closed in the right temporal area, and increased theta- 2 rhythm with eyes open in the left temporal area $(F(5.39)=8.81 ; P<0.00007$; adjusted $\mathrm{R}$-squared $=0.57$ ).

Conclusion: Our findings indicate that $54 \%$ of the patients suffer from postoperative cognitive decline associated with increased theta and decreased alpha rhythms 5-7 years after CABG.

Keywords: Cognitive Dysfunction. Coronary Artery Bypass. Alpha Rhythm. Neurophysiological Tests. Postoperative Cognitive Complications.

\begin{tabular}{llll}
\hline Abbreviations, acronyms \& symbols & & \\
\hline ACEi & Angiotensin-converting enzyme inhibitor & IAF & $=$ Individual alpha frequency \\
ANOVA & $=$ Analysis of variance & Me & $=$ Median \\
BDI-II $\quad=$ Beck Depression Inventory II & MMSE & $=$ Mini-Mental State Examination \\
CABG & $=$ Coronary artery bypass grafting & MSCT & $=$ Multi-slice spiral computed tomography \\
CAD & $=$ Coronary artery disease & NYHA & $=$ New York Heart Association \\
CCB & $=$ Calcium channel blockers & POCD & $=$ Postoperative cognitive dysfunction \\
CSI & $=$ Cognitive status index & S.E. & $=$ Standard error \\
EEG & $=$ Electroencephalography & USA & $=$ United States of America \\
FAB & $=$ Frontal Assessment Battery & & \\
\hline
\end{tabular}

'Research Institute for Complex Issues of Cardiovascular Diseases, Kemerovo, Russia.

This study was carried out at the Research Institute for Complex Issues of Cardiovascular Diseases, Kemerovo, Russia.

The study was carried out under the Program of Fundamental Research of the Siberian branch of the Russian Academy of Sciences.
Correspondence Address:

Irina V. Tarasova

(iD https://orcid.org/0000-0002-6391-0170

Laboratory of Neurovascular Pathology, Research Institute for Complex Issues of Cardiovascular Diseases

Boulevard Sosnovyi 6, Kemerovo, Russian Federation

Zip Code: 650002

E-mail: iriz78@mail.ru 


\section{INTRODUCTION}

Coronary artery disease (CAD) remains the leading cause of death worldwide. Cognitive impairment is common in patients with CAD. These patients are the most difficult ones to treat, particularly those who undergo cardiac surgery ${ }^{[1]}$. Postoperative cognitive dysfunction (POCD) is a decline in cognitive functions following surgery, characterized by impairment of attention, concentration, and memory that may have long-term implications ${ }^{[2]}$. Recent studies have demonstrated that early POCD occurs in almost $70 \%$ of patients undergoing coronary artery bypass grafting (CABG). One year after surgery, cognitive decline occurs in 30-50\% of cases, affecting their long-term prognosis $\left.{ }^{[1,3}\right]$. Recent studies have reported that cognitive disorders may progress from mild cognitive impairment to dementia during a five-year period ${ }^{[4,5]}$. Persistent POCD affects the quality of life, leading to disability and social maladjustment ${ }^{[5,6]}$. Moreover, it is associated with an increased risk of mortality during a seven-year follow-up ${ }^{[6]}$.

The neurophysiological mechanisms underlying the development of POCD are still not clear. A significant proportion of CABG patients are middle-aged or older adults, who suffer from chronic brain ischemia and are more vulnerable to cognitive decline after surgery ${ }^{[1]}$. In addition, surgically-induced brain damage may contribute to the cumulative brain atrophy ${ }^{[7]}$.

The detection of minimal or subclinical signs of brain dysfunction following CABG is still under debate. Quantitative electroencephalography (EEG) is commonly used for the assessment of both normal brain functioning and the development of brain pathology ${ }^{[8-11]}$. Prior studies have shown the relationship between EEG abnormalities and cognitive impairment ${ }^{[8,10,11]}$. A slowing down of resting-state $E E G$ has been reported to be a marker of cortical dysfunction ${ }^{[3,10,11]}$. Bonanni et al. ${ }^{[1]]}$ have found that patients with Parkinson's disease experiencing cognitive impairment and Lewy body dementia demonstrate higher power values of theta activity than healthy control subjects. However, little is known about EEG changes in patients after on-pump $\mathrm{CABG}^{[3]}$. The longterm neurophysiological effects following surgical myocardial revascularization are poorly understood. Moreover, little is known about the structure of cognitive impairment during the long-term postoperative period and the corresponding functional activity of the brain. To facilitate and improve the diagnostic accuracy, detailed neuropsychological examination using the multichannel digital EEG and psychometric tests may be used to detect the long-term brain changes associated with postoperative cognitive impairment.

Therefore, our study aims to evaluate the neurophysiological outcomes of patients 5-7 years after CABG.

\section{METHODS}

\section{Participants}

Forty-five consecutive patients with CAD were recruited to this prospective study in the period from 2010 to 2012. This study was conducted in accordance with the principles of the Helsinki Declaration and was approved by the Institute's Local
Ethics Committee (protocol number no 23 issued on February 02, 2011). The inclusion criteria were as follows: male sex, aged 45-70 years, undergoing elective on-pump CABG, and obtained written informed consent to participate in the study. The exclusion criteria were as follows: diagnosis of neurological or psychiatric disorders that may interfere with cognition; patients with severe depression (score of $\geq 8$ on the Beck Depression Inventory) ${ }^{[12]}$; severe comorbidities; abuse of alcohol or psychotropic drugs; seriously reduced eyesight or other sensory deficits that could affect the assessment of cognitive status; and Mini-Mental State Examination (MMSE) score of $\leq 24$ and Frontal Assessment Battery (FAB) score of $\left.\leq 11^{[13,14}\right]$. Women and patients older than 70 years were excluded from the study in order to avoid the potential effect of the gender and age on the results of the study.

\section{Clinical Assessment}

Patients underwent general medical, neurological, and instrumental examination before CABG and 5-7 years after CABG (January 2016 to December 2017). The examiners were blinded to the patients' cognitive statuses. The severity of coronary artery lesions before CABG was assessed using coronary angiography (Innova 3100; GE Medical Systems, Carrollton, Texas, United States of America [USA]) and the SYNTAX Score calculator (http://www.syntaxscore.com/calculator/start.htm) ${ }^{[15]}$. Carotid artery ultrasound and echocardiography were performed with the Vivid 7 ultrasound machine (GE Medical Systems). Multislice spiral computed tomography (MSCT) was performed with the SIEMENS SOMATOM Sensation 64 (Germany) to detect any nervous system abnormalities. The width of the third ventricle and the ventriculocranial index (Evans index) were measured. Any occurrence of leukoaraiosis, cysts, and gliosis was recorded.

The pre and postoperative clinical and demographic data of patients and prescribed medical therapy are presented in Table 1.

\section{Surgical Procedure}

On-pump CABG was performed under standard perfusion conditions with normothermia and intravenous anesthesia (propofol). The mean cardiopulmonary bypass time was 100.2 \pm 28.24 min, and the aortic cross-clamping time was $62.8 \pm 16.86 \mathrm{~min}$. The number of grafts was $2.6 \pm 0.71$. Invasive hemodynamic control was performed throughout the surgery. Neither episodes of hypotension, nor oxygenation impairment were observed according to the cerebral oximetry (INVOS-3100, SOMANETICS, USA).

\section{Neuropsychological Assessment}

MMSE and FAB were used for cognitive screening. Neuropsychological testing included the assessment of psychomotor and executive function, attention, and short-term memory using the neuropsychological test battery from the psychophysiological complex software Status PF. The detailed description of the neuropsychological tests is shown in Table 2. Postoperative cognitive decline five years after CABG was determined by a $20 \%$ decrease in the cognitive score compared to baseline in $20 \%$ of the tests ${ }^{[16,17]}$. The integral cognitive status index (CSI) was calculated by determining the average distance 
Table 1 - Clinical and demographic characteristics of patients before CABG and 5-7 years after CABG.

\begin{tabular}{|c|c|c|}
\hline \multirow[b]{2}{*}{ Variable } & \multicolumn{2}{|c|}{ Patients, $n=45$} \\
\hline & $\begin{array}{l}\text { Before } \\
\text { CABG }\end{array}$ & $\begin{array}{l}\text { 5-7 years after } \\
\text { CABG }\end{array}$ \\
\hline Age, years, Me (Q25; Q75) & $56.0(52 ; 59)$ & $62.5(58,5 ; 66)$ \\
\hline $\begin{array}{l}\text { Educational attainment, } \mathbf{n}(\%) \\
8 \text { years } \\
10-12 \text { years } \\
15 \text { years }\end{array}$ & $\begin{array}{c}2(4 \%) \\
31(69 \%) \\
12(27 \%)\end{array}$ & - \\
\hline Left ventricular ejection fraction, \%, Me (Q25; Q75) & $60.0(52 ; 63)$ & $60.5(54 ; 63)$ \\
\hline $\begin{array}{l}\text { Functional class angina, } \mathbf{n}(\%) \\
0 \\
|-| \mid \\
\text { III }\end{array}$ & $\begin{array}{c}0(0 \%) \\
30(77 \%) \\
15(33 \%)\end{array}$ & $\begin{array}{c}32(71 \%) \\
12(27 \%) \\
1(2 \%)\end{array}$ \\
\hline $\begin{array}{l}\text { Functional class NYHA, } \mathbf{n}(\%) \\
\qquad|-| \mid \\
I \|\end{array}$ & $\begin{array}{l}37(82 \%) \\
8(18 \%)\end{array}$ & $\begin{array}{l}44(98 \%) \\
1(2 \%)\end{array}$ \\
\hline $\begin{array}{l}\text { Severity of coronary arteries lesions by the SYNTAX scale before surgery, scores, Me (Q25; } \\
\text { Q75) }\end{array}$ & $22.5(16.5 ; 29.3)$ & - \\
\hline History of myocardial infarction, n (\%) & $6(13 \%)$ & - \\
\hline Myocardial infarction after surgery, n (\%) & - & $1(2 \%)$ \\
\hline Stenoses of the carotid arteries, n (\%) & $21(47 \%)$ & $23(51 \%)$ \\
\hline Diabetes mellitus Type 2, n (\%) & $10(22 \%)$ & $10(22 \%)$ \\
\hline $\begin{array}{l}\text { Cardiac rate disturbance and disorder of conduction, } \mathbf{n}(\%) \\
\text { Atrial fibrillation } \\
\text { Ventricular contractions, III-IV Lown grade }\end{array}$ & $\begin{array}{l}0(0 \%) \\
0(0 \%)\end{array}$ & $\begin{array}{l}4(9 \%) \\
7(16 \%)\end{array}$ \\
\hline $\begin{array}{l}\text { Medication, n (\%) } \\
\text { ACEi } \\
\text { Statin } \\
\text { Beta-blockers } \\
\text { Antiplatelet drugs } \\
\text { CCB } \\
\text { Nitrates }\end{array}$ & $\begin{array}{c}40(89 \%) \\
30(67 \%) \\
44(98 \%) \\
43(96 \%) \\
19(42 \%) \\
7(16 \%)\end{array}$ & $\begin{array}{c}20(44 \%) \\
31(69 \%) \\
37(82 \%) \\
42(93 \%) \\
0(0 \%) \\
1(2,2 \%)\end{array}$ \\
\hline MMSE, scores, Me (Q25; Q75) & $28(26 ; 28)$ & $28(27 ; 29)$ \\
\hline FAB, scores, Me (Q25; Q75) & $17(16 ; 17)$ & $16(15 ; 17)$ \\
\hline BDI-II, scores, Me (Q25; Q75) & $2(1 ; 3)$ & $3(2 ; 5)$ \\
\hline
\end{tabular}

$\mathrm{ACE} i=$ angiotensin-converting enzyme inhibitor; BDI-II=Beck Depression Inventory II; CABG=coronary artery bypass grafting; $\mathrm{CCB}=$ calcium channel blockers; FAB=Frontal Assessment Battery; Me=median; MMSE=Mini-Mental State Examination; NYHA=New York Heart Association

from the patient's values to the reference ones using the following formula:

$$
C S I=1-\frac{\sqrt{(1-Y 1)^{2}+(1-Y 2)^{2}+(1-Y 3)^{2}+(1-Y 4)^{2}+(1-Y 5)^{2}}}{5}
$$

where:

$Y$ is a recording value of the cognitive indicator; $Y 1$ is the mean value of the reaction time in psychomotor and executive function tests; $Y 2$ is the mean value of the errors in psychomotor and executive function tests; $Y 3$ is the mean value of the missing signals in psychomotor and executive function tests; $Y 4$ is the mean value of the short-term memory indicator; and $Y 5$ is the mean value of the attention indicator.

\section{EEG Recording}

A monopolar EEG in 62 channels (bandwidth 0.1-50.0 Hz), in accordance with the international 10-20 system, with the 
Table 2. Cognitive test battery for assessing cognitive function in CABG patients.

\begin{tabular}{|c|c|}
\hline Cognitive tests and indicators & Description of the procedure \\
\hline $\begin{array}{l}\text { Complex visual-motor reaction } \\
\text { Reaction time, ms } \\
\text { Errors, } n\end{array}$ & $\begin{array}{l}\text { Reaction latencies of the right and left hands to stimuli (different } \\
\text { colors of rectangles) when the subject should choose one of the } \\
\text { three presented signals (the number of signals in the test is 30) }\end{array}$ \\
\hline $\begin{array}{l}\text { Level of functional mobility of nervous processes } \\
\text { responses to "feedback" } \\
\text { Reaction time, ms } \\
\text { Errors, } n \\
\text { Missed signals, n }\end{array}$ & $\begin{array}{l}\text { The previous test is conducted in the feedback mode. The } \\
\text { duration of the exposure to the test signal (see above) is changed } \\
\text { automatically; the exposure of the next signal is shortened by } 20 \\
\text { ms with each correct answer and extended by } 20 \text { ms, if the answer } \\
\text { is wrong (the number of signals in the test is 120) }\end{array}$ \\
\hline $\begin{array}{l}\text { Performance of the brain responses to "feedback" } \\
\text { Reaction time, ms } \\
\text { Errors, } n \\
\text { Missed signals, } n\end{array}$ & $\begin{array}{l}\text { The previous test is conducted in the feedback mode for a fixed } \\
\text { period ( } 5 \text { min). It is necessary to process the maximum number of } \\
\text { signals presented with a given exposure }\end{array}$ \\
\hline $\begin{array}{l}\text { The Bourdon's test } \\
\text { Processed symbols per } 1 \text { min, } \mathrm{n} \\
\text { Processed symbols per } 4 \text { mins, } \mathrm{n}\end{array}$ & $\begin{array}{l}\text { The subject is provided with the alphabetic version of the } \\
\text { Bourdon's test to highlight certain letters for the lead time of } 4 \text { mins }\end{array}$ \\
\hline 10 words memorizing test, $n$ & $\begin{array}{l}\text { To remember as many of } 10 \text { words presented one after another as } \\
\text { possible }\end{array}$ \\
\hline 10 numbers memorizing test, $\mathrm{n}$ & $\begin{array}{l}\text { To remember as many of } 10 \text { numbers presented one after another } \\
\text { as possible }\end{array}$ \\
\hline 10 nonsense syllable memorizing test, $\mathrm{n}$ & $\begin{array}{l}\text { To remember as many of } 10 \text { nonsense syllables presented one after } \\
\text { another as possible }\end{array}$ \\
\hline
\end{tabular}

$\mathrm{CABG}=$ coronary artery bypass grafting

eyes closed and open was recorded with an amplifier (Neuvo SynAmps2, Compumedics, Charlotte, North Carolina, USA) using a modified 64-channel cap (QuikCap; Neurosoft, El Paso, Texas, USA). The reference electrode was attached to the tip of the nose, and the ground electrode to the forehead (impedance $<5 \mathrm{k} \Omega$ ). The recording duration was 10 min (five minutes with the eyes closed). Data were analyzed offline, and a visual search for recording artifacts was conducted. Artifact-free EEG fragments were divided into 2-s epochs and subjected to the Fourier transform. The peak individual alpha frequency (IAF) for each patient was determined. Because the mean IAF of the study group was $9.4 \mathrm{~Hz}$, we used standard frequency bands. For each patient, the EEG power values were averaged within theta-1 $(4-6 \mathrm{~Hz})$, theta-2 $(6-8 \mathrm{~Hz})$, alpha-1 $(8-10 \mathrm{~Hz})$, alpha-2 (10-13 Hz), beta-1 (13-20 Hz), and beta-2 (20-30 Hz) ranges. Further, the EEG power values recorded at nearby electrode sites were summarized in five areas in the left and right hemispheres:

1. Frontal (Fp1/2+AF3/4+F1/2+Fp3/4+Fp5/6+F7/8);

2. Central $(\mathrm{FC} 1 / 2+\mathrm{FC} 3 / 4+\mathrm{FC} 5 / 6+\mathrm{C} 1 / 2+\mathrm{C} 3 / 4+\mathrm{C} 5 / 6)$;

3. Temporal (FT7/8+T7/8+TP7/8);

4. Parietal $(\mathrm{CP} 1 / 2+\mathrm{CP} 3 / 4+\mathrm{CP} 5 / 6+\mathrm{P} 1 / 2+\mathrm{P} 3 / 4+\mathrm{P} 5 / 6+\mathrm{P} 7 / 8)$;

5. Occipital (PO3/4+PO5/6+PO7/8+O1/2).

The parameters recorded at the midline (Fpz, Fz, etc.) were excluded from the analysis.

\section{Statistical Analyses}

Statistical analysis was performed using the software STATISTICA 10.0 (StatSoft, Tulsa, Oklahoma, USA). Variable distributions were examined using scatterplots/histograms and normality tests. Continuous variables are expressed as medians and quartiles, and categorical variables are expressed as numbers and percentages. Two-tailed Mann-Whitney $U$ and Wilcoxon tests were used for continuous variables. Continuity-corrected $x^{2}$ or Kruskal-Wallis tests were used for categorical variables. Nonnormally distributed EEG power values were log-transformed. Repeated-measures analyses of variance (ANOVAs) were used to analyze EEG power for the abovementioned frequency ranges and for the eyes-open and eyes-closed resting states, with GROUP (patients with and without postoperative cognitive decline), TIME OF STUDY (before CABG, five years after CABG), AREA (frontal, central, temporal, parietal, occipital), and LATERALITY (left and right hemispheres) as within-subject factors. The GreenhouseGeyser correction of statistical significance was performed. A subsequent statistical significance analysis was performed using post-hoc and Newman-Kels tests. The significance level was 5\%.

To identify correlations between EEG and neuropsychological test data, the Pearson's correlation analysis was performed. To correct the multiplicity of comparisons, the significance level was 
increased up to $P \leq 0.01$. To identify demographic, clinical, and EEG variables associated with adverse cognitive outcomes, the multiple linear regression with a stepwise approach was applied. Independent variable selection was based on our previous research on $P O C D$ in $C A D$ patients, the characteristics of the study population, and the correlation analysis data described ${ }^{[1,3]}$. CSI was the dependent variable.

\section{RESULTS}

\section{Clinical and Neuropsychological Data}

There were no significant adverse cardiovascular events among patients in the early and long-term postoperative period of CABG (the mean observation period was 6.5 years). One patient had myocardial infarction and underwent percutaneous coronary intervention. The details are provided in Table 1.

Serial MSCT reported specific morphological changes in the brain tissue. The third ventricle significantly expanded from $7.1 \pm 2.0 \mathrm{~mm}$ to $8.6 \pm 2.4 \mathrm{~mm}(P=0.04)$. The number of patients with cysts and gliosis (3 [6.7\%] vs.. 12 [26.7\%], $P=0.0001)$ and the number of patients with leukoaraiosis (13 [29\%] vs. 36 [81\%], $P=0.0001)$ increased in the long-term period. The Evans index did not change significantly ( 29 vs. $31, P=0.8$ ).

The incidence of late postoperative cognitive decline was 54\% (24 patients). The structure of postoperative cognitive decline consisted of impaired neurodynamic function (70\%, 30 patients), memory (47\%, 21 patients), and attention disorders (21\%, nine patients). The number of patients without impairment was $9 \%$ (four patients).

\section{EEG Data Analysis}

The ANOVA revealed significant interactions associated with postoperative cognitive decline in the theta and alpha frequency ranges.

For the theta-1 power values with the eyes closed, the following significant interactions were found: TIME $\times$ AREA - $\left(F_{(4,172)}=13.87, P=0.000\right)$, TIME $\times$ AREA $\times$ LATERALITY $-\left(F_{(4,}\right.$ $\left.\left.{ }_{172}\right)=6.56, P=0.0005\right)$, and GROUP $\times$ TIME $\times$ AREA $\times$ LATERALITY - $\left(F_{(4,172)}=2.89, P=0.04\right)$. Five to seven years after $C A B G$, patients demonstrated a significant increase in the theta-1 power compared to baseline in the frontal and temporal areas, with more pronounced changes in the right hemisphere. Baseline and postoperative theta-1 power did not differ between the groups. However, patients with postoperative cognitive decline exhibited significantly increased theta- 1 power in the frontal and temporal left and right hemispheres, whereas patients without cognitive decline demonstrated its increase in the temporal regions of the right hemisphere (Figure 1).

For the alpha-1 range, with the eyes closed, the following significant interactions were found: TIME $\times$ AREA $-F_{(4,172)}=5.62$, $P=0.005)$ and GROUP $\times$ TIME $\times$ LATERALITY $-\left(F_{(1,43)}=4.80, P=0.03\right)$. All patients demonstrated a decrease in the alpha-1 power compared to baseline in the parietal and occipital cortices. Between-group differences were found in the left hemisphere. Before surgery, the alpha-1 power was higher in the group of patients without cognitive decline compared to patients with postoperative cognitive decline. At the follow-up, only patients without cognitive decline demonstrated a significant decrease in the alpha-1 power in the left hemisphere (Figure 2). There were no significant changes found for the right hemisphere.

For the alpha-1 range, with the eyes open, the factor of TIME $-\left(F_{(1,43)}=4.65, P=0.04\right)$ interacted with TIME $\times$ AREA $-\left(F_{(4,}\right.$ $\left.{ }_{172)}=4.96, P=0.006\right)$ and GROUP $\times$ TIME $\times$ AREA $\times$ LATERALITY - $\left(F_{(4,172)}=5.07, P=0.0025\right)$. All patients exhibited increased alpha-1 power compared to baseline. Significant changes were found in the occipital and temporal cortices, which were more pronounced in the group of patients with cognitive decline.

\section{Correlation Analysis}

The results of the correlation analysis are presented in Figure 3. The number of memorized numbers negatively correlated with theta-1 power with the eyes closed in the left parietal cortex. The number of errors during the brain performance test directly correlated with the theta- 2 and alpha- 1 rhythm power with the eyes closed in the right hemisphere. In addition, the number of missed signals negatively correlated with the theta-2 power in the posterior brain regions (left and right hemispheres). Negative correlations between the attention and theta-2 power with the eyes open in all areas of the left hemisphere were found.

\section{Multiple Regression Analyses}

The first multiple regression model was generated based on clinical and demographic variables. We found that the time of cardiopulmonary bypass had the highest prognostic weight (Beta coefficient=-0.35; $P=0.0003$ ). The estimated regression standardized coefficients were as follows: $P=0.41$; $R$-squared $=0.17$; adjusted R-squared $=0.09 ; F_{(3.41)}=2.28 ; P=0.09$. The standard estimation error was 0.137 . However, this model had a low prognostic value.

The next regression model was generated with a compilation of clinical variables and EEG data. The theta-2 power had the greatest prognostic weight along with the mean alpha frequency (Table 3). The estimated regression standardized coefficients of the model were as follows: $R=0.80$; R-squared $=0.64$; adjusted R-squared $=0.57 ; F_{(5.39)}=8.81 ; P<0.00007$. The standard estimation error was 0.09 . Slower mean alpha frequency, decreased theta-2 rhythm with the eyes closed in the right temporal area and sequential organ failure assessment score, increased theta-2 rhythm with the eyes open in the left temporal area and theta-2 rhythm with the eyes closed in the right frontal area were the independent predictors of cognitive decline. These predictors explained $57 \%$ of the variance of the CSI scores.

\section{DISCUSSION}

We found that $54 \%$ of patients demonstrated a decline in cognitive function compared to baseline during the long-term postoperative period of CABG (5-7 years).

The mechanisms underlying the development of POCD are still poorly understood. Some researchers have reported that cerebral microembolic load, inflammatory response, and nonphysiological perfusion of cardiopulmonary bypass contribute 


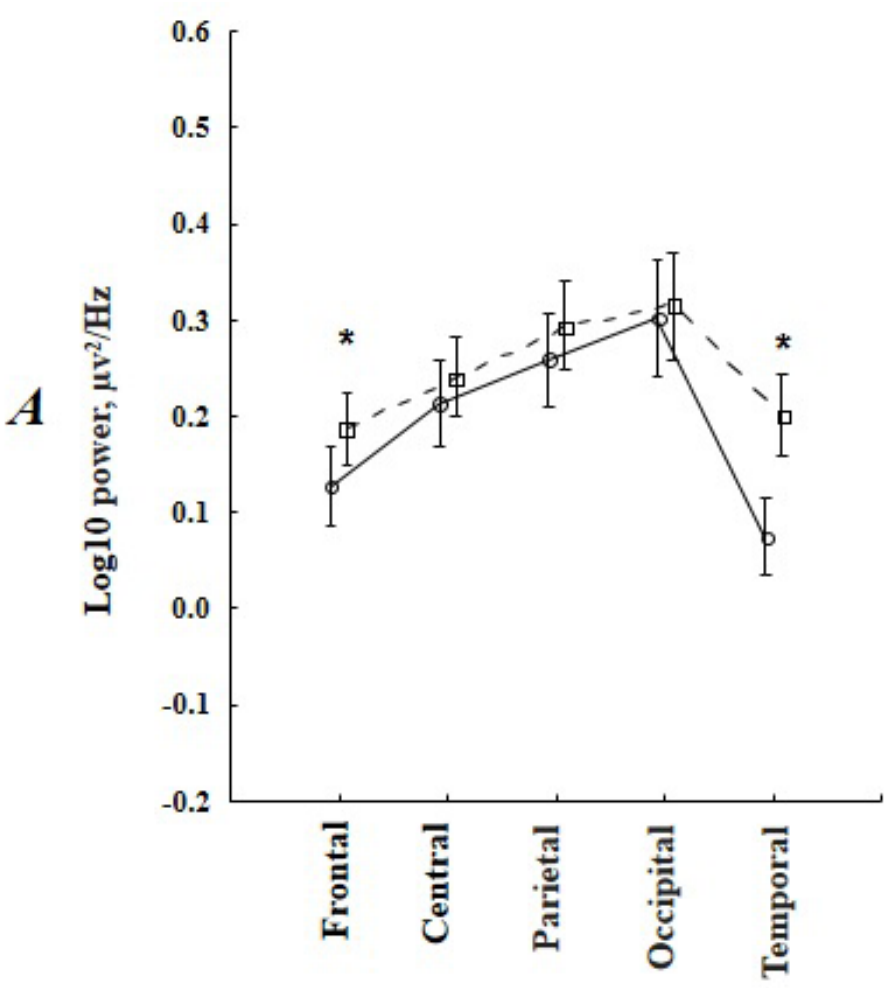

Left hemisphere

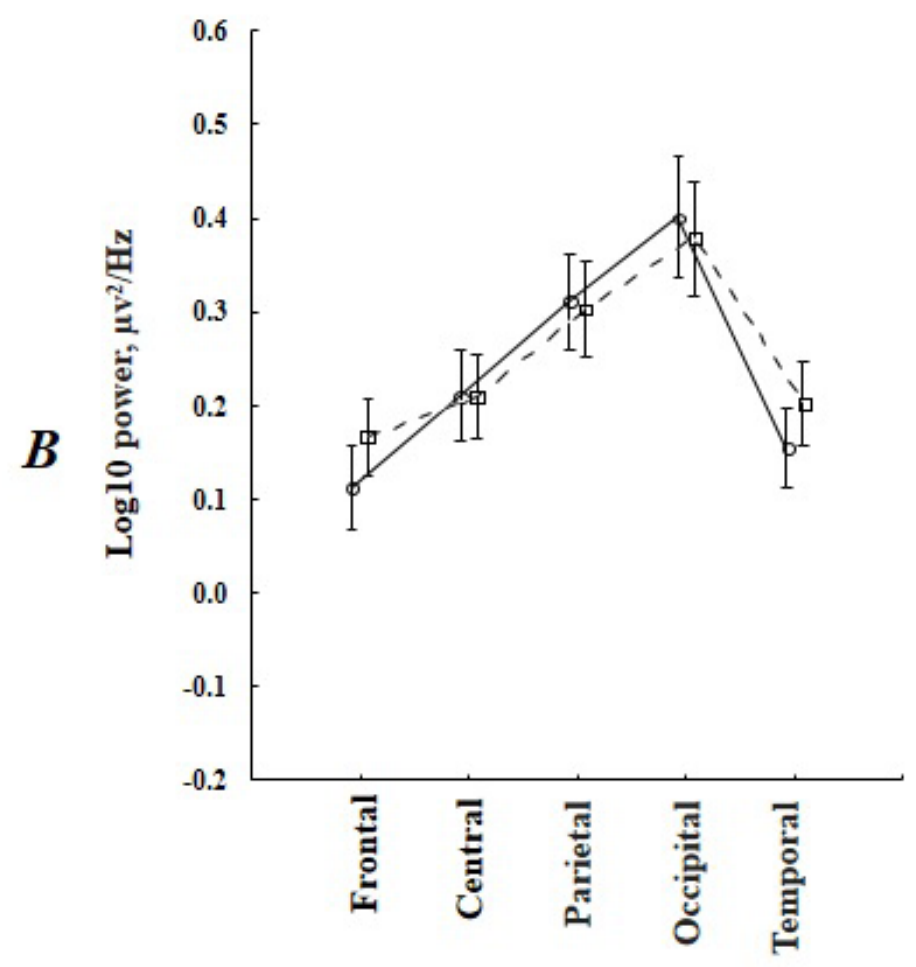

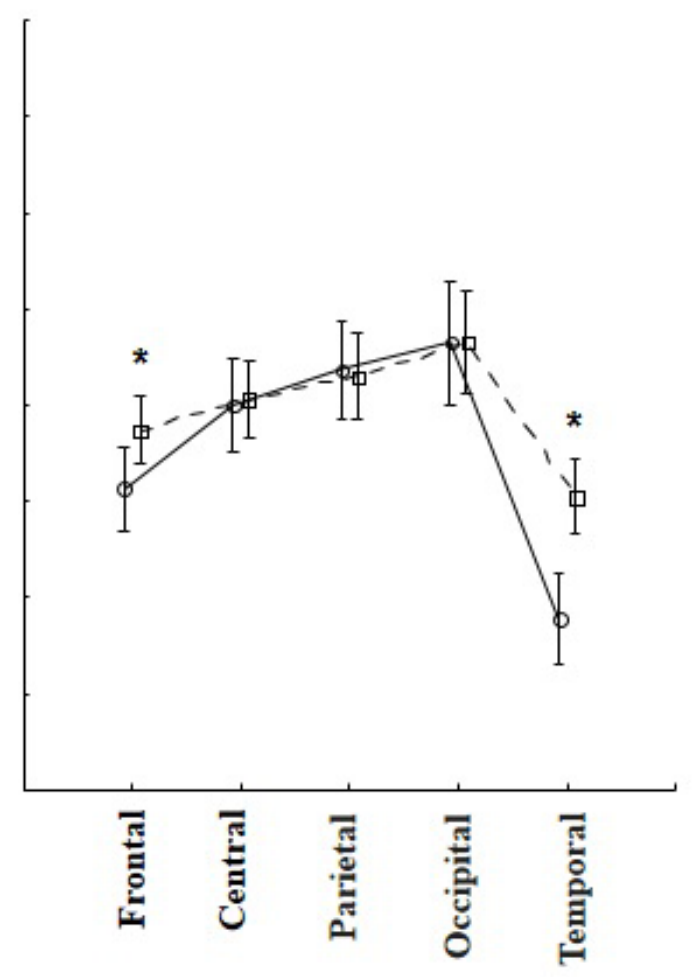

Right hemisphere

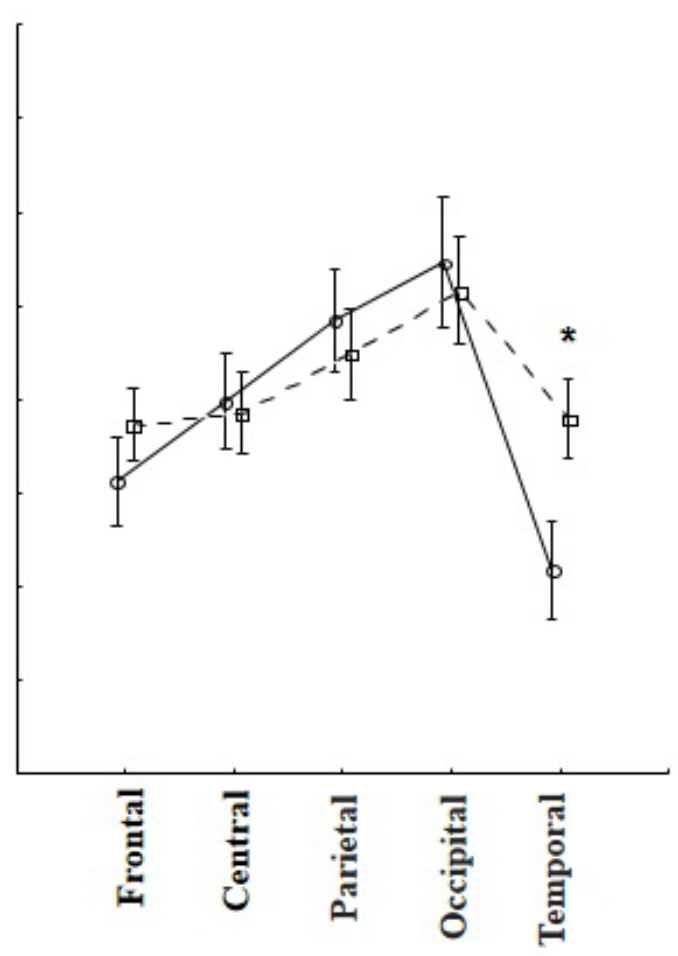

Fig. 1 - Topographic changes of the theta-1 rhythm power with eyes closed in patients with cognitive decline (A) and without cognitive decline (B) in the long-term period after coronary artery bypass grafting. Solid lines indicate preoperative indicators, dashed lines indicate 5-7 years after surgery. ${ }^{*}$ marked significant differences $(P \leq 0.05)$. 


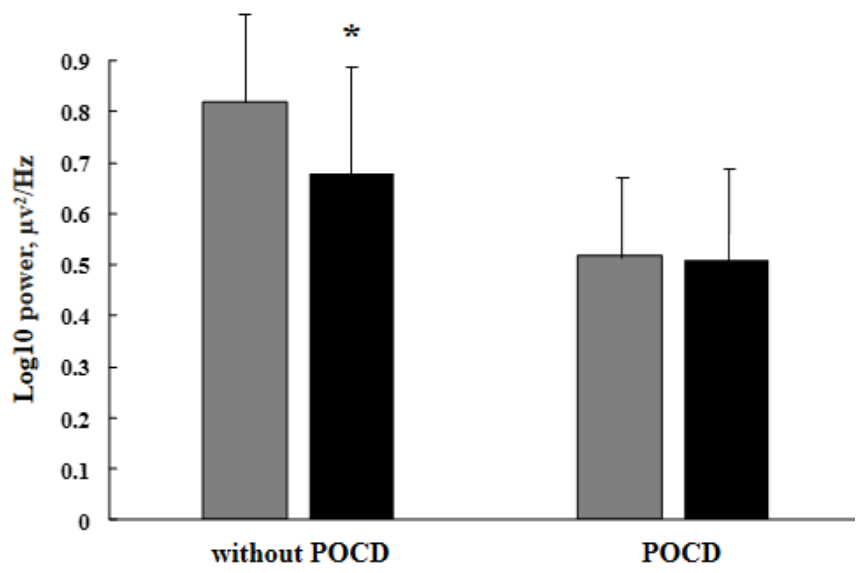

Fig. 2 - Changes of alpha-1 rhythm power with eyes closed in the left hemisphere in patients depending on the cognitive decline in the long-term period after coronary artery bypass grafting. Gray columns indicate preoperative indicators, black columns indicate 5-7 years after surgery. ${ }^{*}$ marked significant differences $(P \leq 0.05)$. $P O C D=$ postoperative cognitive dysfunction to postoperative cognitive impairment in early postoperative period and late cognitive recovery ${ }^{[2,17]}$. However, Selnes et al. ${ }^{[16]}$ have proposed that preexisting cerebrovascular disorders more likely contribute to long-term POCD rather than cardiopulmonary bypass.

Our data have shown that long-term postoperative cognitive decline is most pronounced in the neurodynamic domains and short-term memory. Notably, neurodynamic test performance allows evaluating executive control. Alosco et al. ${ }^{[18]}$ have found that patients with cardiovascular diseases exhibit decreased memory and executive functions. Patients with cardiovascular diseases are highly susceptible to ischemic changes in the frontal brain regions, resulting in executive function impairment ${ }^{[19,20]}$. Additionally, short-term memory decline has been found in patients with heartfailure ${ }^{[21,22]}$. Ourfindings are consistent with the abovementioned studies, but these neurophysiological results have been obtained in a specific sample of patients undergoing CABG. The factors associated with cardiac surgery affect brain functions, and their effects are particularly pronounced in the early postoperative CABG period. However, less is known about their long-term impact. Researchers have suggested that brain
A

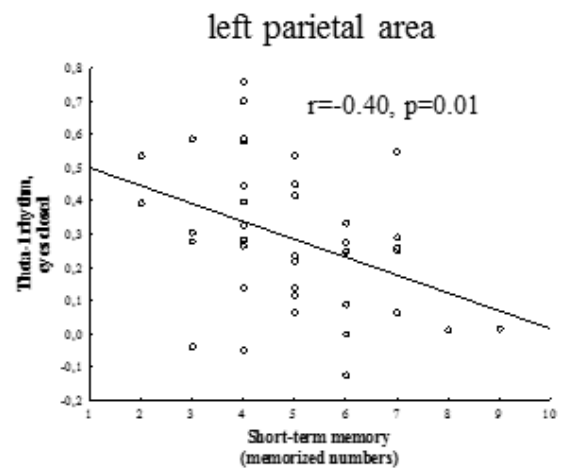

left frontal area

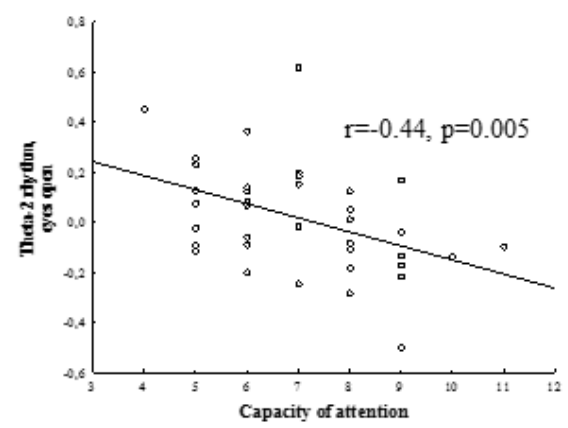

right parietal area

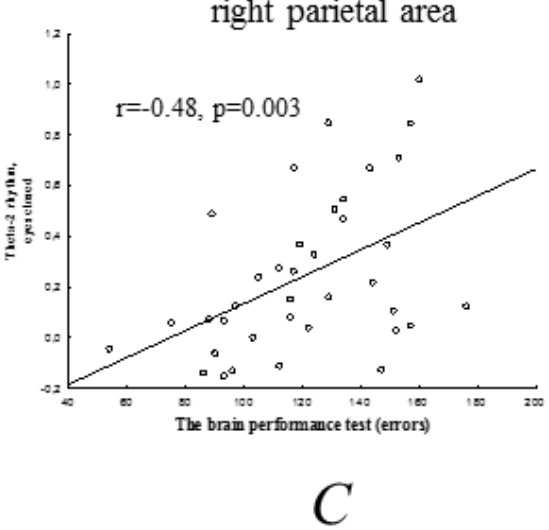

left central area

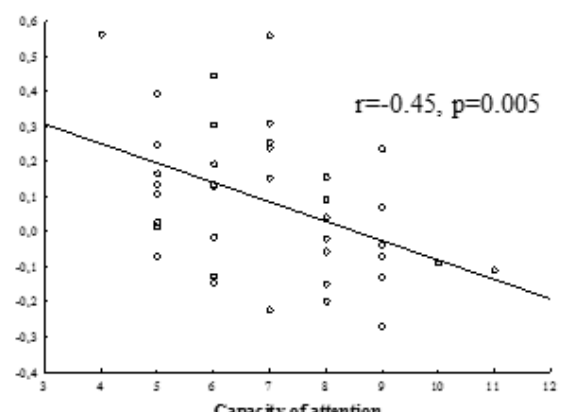

$B$

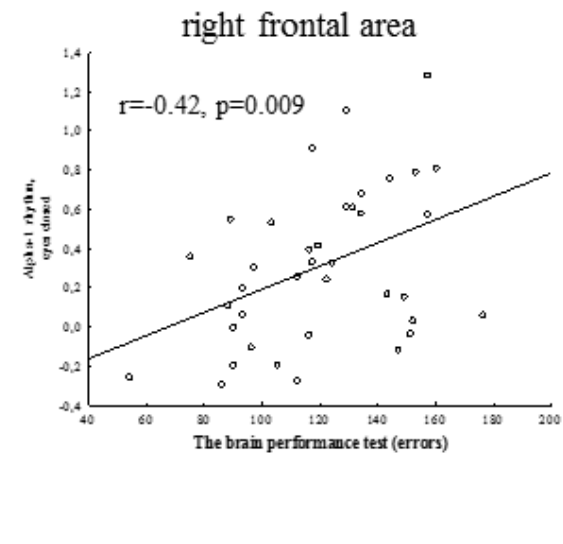

left temporal area

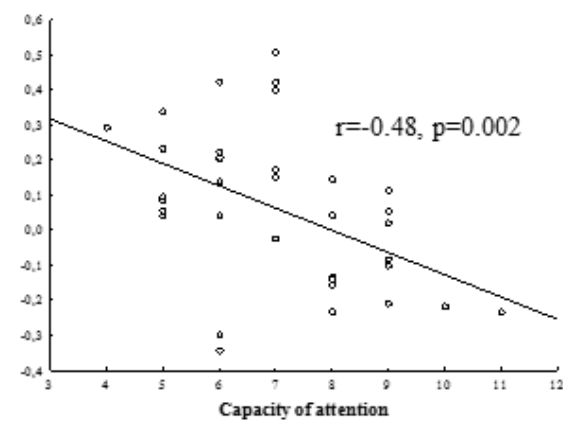

Fig. 3 - Correlations between the cognitive status indicators and electroencephalographical power: (A) a correlation between number of memorized digit and log-transformed theta-1 rhythm power values (eyes closed); (B) correlations between errors in the brain performance test and log-transformed theta-2 and alpha-1 rhythms power values (eyes closed); and (C) correlations between a capacity of attention and log-transformed theta-2 rhythm power values (eyes open) in the coronary artery disease patients. 
Table 3. Results of the regression analysis of factors associated with postoperative cognitive decline five years after CABG in CAD patients.

\begin{tabular}{l|c|c|c|c}
\hline Dependent variable - integral cognitive status index (or CSI) & Beta & S.E. - Beta & t & P-value \\
\hline Intercept & & & -2.335 & 0.028 \\
\hline Mean alpha frequency & 0.669 & 0.133 & 5.025 & 0.00004 \\
\hline Theta-2 rhythm with eyes closed in right temporal area & 1.134 & 0.258 & 4.394 & 0.0002 \\
\hline Theta-2 rhythm with eyes open in left temporal area & -0.600 & 0.162 & -3.711 & 0.001 \\
\hline Sequential organ failure assessment score & 0.296 & 0.131 & 2.268 & 0.03 \\
\hline Theta-2 rhythm with eyes closed in right frontal area & -0.294 & 0.219 & -1.338 & 0.19 \\
\hline
\end{tabular}

$\mathrm{CABG}=$ coronary artery bypass grafting; $\mathrm{CAD}=$ coronary artery disease; S.E. $=$ standard error

damage in the early postoperative CABG may underlie the development of persistent cognitive deficit $[1,2,6,17]$.

We have found that cognitive deficit is accompanied with the morphological changes in brain tissue and an increase in the background theta activity with the eyes closed and alpha activity with the eyes open in the long-term period after CABG. The resting-state alpha activity with closed eyes has decreased compared to preoperative indicators. Previous studies have shown that increased lower frequencies, reduced complex activities, and incoherent cortical regions/fast rhythms recorded on the electroencephalogram may indicate $P O C D^{[3,23]}$. We may suggest that $C A B G$ patients suffer from insufficient cerebral circulation, causing neuronal dysfunction, in late postoperative period. As a result, a negative shift of the EEG rhythms occurs. Kramberger et al. ${ }^{[24]}$ demonstrated that the dominant background EEG activity slowing is correlated with an increased T-tau protein and a lower A $42 /$ P-tau ratio in cerebrospinal fluid. The atrophic changes in the brain, such as an expansion of the brain ventricular system and leukoaraiosis, can be regarded as clinical manifestations of cerebral microangiopathy ${ }^{[25]}$. In our study, almost $50 \%$ of patients suffered from carotid stenosis and 9\% had postoperative atrial fibrillation. These comorbidities could promote pathologic cardiac remodeling and contribute to the brain ischemia.

Pathological rearrangements of brain oscillations are associated with altered regulation, reduced-complexity oscillatory processes, and dysfunctions of neurotransmission ${ }^{[26]}$. It has also been hypothesized that the diminished predominance of posterior alpha activity over slow activity (theta) and an increase in theta activity relative to alpha activity may be associated with impaired regulation of the cholinergic basal forebrain. This could cause prolonged agitation in the stem cholinergic pathways ${ }^{[8]}$.

Thus, the combination of EEG resting-state characteristics can indicate an imbalance between cortical and subcortical structures, and a decrease in the functional activity of the cortex ${ }^{[27,28]}$. Topographically identified changes in the background EEG activity were localized within the frontal, temporal, and parieto-occipital regions. The results of the correlation and multiple regression analyses confirmed the involvement of these brain structures in the process of cognitive decline in the late postoperative period. Thus, the poorest short-term memory values corresponded to large values of background theta- 1 activity with the eyes closed in the left parietal cortex. Neurodynamic disorders were associated with impaired function of the right parietal-occipital regions of the cortex. Neurodegeneration primarily affects the hippocampus and adjacent brain regions, i.e., the cingulate and parietaltemporal cortex ${ }^{[29,30]}$. Further, the recent studies of cognitive disorders in the cohort of elderly patients with cardiovascular diseases have indicated certain difficulties in the differentiation of the neurodegenerative and ischemic patterns of brain damage; rather, we should refer to a mixed etiology of cognitive deficit ${ }^{[19,31]}$.

The data obtained in this study confirm this assumption. Since EEG signs of cerebral dysfunction in the late period of CABG are localized in the frontal and parieto-temporal brain regions, cognitive decline of executive functions and shortterm memory is found. However, the factors contributing to late cognitive decline after CABG remain unclear. The high incidence of cognitive decline in the long-term postoperative period and the ambiguity of the underlying mechanisms prompt further study of this phenomenon in specific groups of CAD patients. Our findings indicate the need to improve approaches to the postoperative follow-up management of patients undergoing cardiac surgery, capable of minimizing the development of adverse neurological outcomes.

\section{Limitations}

The findings of our study should be interpreted within the context of its limitations. We did not recruit a control group of healthy volunteers to assess age-related changes in this group of patients. We have a small sample of patients ( $n=45)$, since we were recruiting only consecutive ones. Additionally, only male CAD patients were included. Therefore, it is unclear whether our findings can be extrapolated to women. We plan to address all of these issues in our future studies. 


\section{CONCLUSION}

Our findings demonstrate that $54 \%$ of the patients suffer from a postoperative cognitive decline associated with increased theta and decreased alpha rhythms 5-7 years after CABG. The long-term postoperative cognitive decline is most pronounced in the neurodynamic domains and short-term memory. This indicates the need to improve approaches to the postoperative follow-up management of patients undergoing cardiac surgery, capable of minimizing the development of adverse neurological outcomes.

\section{ACKNOWLEDGMENTS}

We thank Olga V. Maleva and Anton V. Solodukhin for expert technical assistance.

\section{No financial support. \\ No conflict of interest.}

\section{Authors' roles \& responsibilities}

IVT Substantial contributions to the acquisition and analysis of data for the work; drafting the work; final approval of the version to be published

OAT Substantial contributions to the acquisition of data for the work; drafting the work; final approval of the version to be published

IDS Substantial contributions to the acquisition of data for the work; final approval of the version to be published

OLB Revising the work critically for important intellectual content; agreement to be accountable for all aspects of the work in ensuring that questions related to the accuracy or integrity of any part of the work are appropriately investigated and resolved; final approval of the version to be published

\section{REFERENCES}

1. Trubnikova OA, Mamontova AS, Syrova ID, Maleva OV, Barbarash OL. Does preoperative mild cognitive impairment predict postoperative cognitive dysfunction after on-pump coronary bypass surgery? J Alzheimers Dis. 2014;42 Suppl 3:S45-51. doi:10.3233/JAD-132540.

2. Yuan SM, Lin H. Postoperative cognitive dysfunction after coronary artery bypass grafting. Braz J Cardiovasc Surg. 2019;34(1):76-84. doi:10.21470/1678-9741-2018-0165.

3. Tarasova IV, Trubnikova OA, Barbarash OL, Barbarash LS. EEG changes in patients with early and long-term postoperative cognitive dysfunction after on-pump coronary artery bypass surgery. Neurol J. 2017;22(3):13641. doi:10.18821/1560-9545-2017-22-3-136-141.

4. Evered L, Scott DA, Silbert B. Cognitive decline associated with anesthesia and surgery in the elderly: does this contribute to dementia prevalence? Curr Opin Psychiatry. 2017;30(3):220-6. doi:10.1097/ YCO.0000000000000321.

5. Sorrell JM. Postoperative cognitive dysfunction in older adults: a call for nursing involvement. J Psychosoc Nurs Ment Health Serv. 2014;52(11):1720. doi:10.3928/02793695-20141021-03.
6. Evered LA, Silbert BS, Scott DA, Maruff P, Ames D. Prevalence of dementia 7.5 years after coronary artery bypass graft surgery. Anesthesiology. 2016;125(1):62-71. doi:10.1097/ALN.0000000000001143.

7. Kline RP, Pirraglia E, Cheng H, De Santi S, Li Y, Haile M, et al. Surgery and brain atrophy in cognitively normal elderly subjects and subjects diagnosed with mild cognitive impairment. Anesthesiology. 2012;116(3):603-12. doi:10.1097/ALN.0b013e318246ec0b.

8. Babiloni C, Lizio R, Marzano N, Capotosto P, Soricelli A, Triggiani Al, et al. Brain neural synchronization and functional coupling in Alzheimer's disease as revealed by resting state EEG rhythms. Int J Psychophysiol. 2016;103:88-102. doi:10.1016/j.jpsycho.2015.02.008.

9. Nobukawa S, Kikuchi M, Takahashi T. Changes in functional connectivity dynamics with aging: a dynamical phase synchronization approach. Neuroimage. 2019;188:357-68. doi:10.1016/j.neuroimage.2018.12.008.

10. Snyder SM, Hall JR, Cornwell SL, Falk JD. Addition of EEG improves accuracy of a logistic model that uses neuropsychological and cardiovascular factors to identify dementia and MCl. Psychiatry Res. 2011;186(1):97-102. doi:10.1016/j.psychres.2010.04.058.

11. Bonanni L, Perfetti B, Bifolchetti S, Taylor JP, Franciotti R, Parnetti L, et al. Quantitative electroencephalogram utility in predicting conversion of mild cognitive impairment to dementia with Lewy bodies. Neurobiol Aging. 2015;36(1):434-45. doi:10.1016/j.neurobiolaging.2014.07.009.

12. Beck AT, Steer RA, Brown GK. Beck Depression Inventory. 2nd ed. New York (NY): Psychological Corporation; 1996.

13. Folstein MF, Folstein SE, McHugh PR. "Mini-mental state". A practical method for grading the cognitive state of patients for the clinician. J Psychiatr Res. 1975;12(3):189-98. doi:10.1016/0022-3956(75)90026-6.

14. Dubois B, Slachevsky A, Litvan I, Pillon B. The FAB: a frontal assessment battery at bedside. Neurology. 2000;55(11):1621-6. doi:10.1212/ wnl.55.11.1621.

15. Carnero-Alcázar M, Maroto Castellanos LC, Silva Guisasola JA, Cobiella Carnicer J, Alswies A, Fuentes Ferrer ME, et al. SYNTAX score is associated with worse outcomes after off-pump coronary artery bypass grafting surgery for three-vessel or left main complex coronary disease. JThorac Cardiovasc Surg. 2011;142(3):e123-32. doi:10.1016/j.jtcvs.2010.10.036.

16. Selnes OA, Gottesman RF, Grega MA, Baumgartner WA, Zeger SL, McKhann GM. Cognitive and neurologic outcomes after coronaryartery bypass surgery. N Engl J Med. 2012;366(3):250-7. doi:10.1056/ NEJMra1100109.

17. Bhamidipati D, Goldhammer JE, Sperling MR, Torjman MC, McCarey MM, Whellan DJ. Cognitive outcomes after coronary artery bypass grafting. J Cardiothorac Vasc Anesth. 2017;31 (2):707-18. doi:10.1053/j. jvca.2016.09.028.

18. Alosco ML, Spitznagel MB, Sweet LH, Josephson R, Hughes J, Gunstad J. Cognitive dysfunction mediates the effects of poor physical fitness on decreased functional independence in heart failure. Geriatr Gerontol Int. 2015;15(2):174-81. doi:10.1111/ggi.12245.

19. Gorelick PB, Counts SE, Nyenhuis D. Vascular cognitive impairment and dementia. Biochim Biophys Acta. 2016;1862(5):860-8. doi:10.1016/j. bbadis.2015.12.015.

20. Hsu CL, Best JR, Davis JC, Nagamatsu LS, Wang S, Boyd LA, et al. Aerobic exercise promotes executive functions and impacts functional neural activity among older adults with vascular cognitive impairment. $\mathrm{Br}$ J Sports Med. 2018;52(3):184-91. doi:10.1136/bjsports-2016-096846.

21. Leto L, Feola M. Cognitive impairment in heart failure patients. J Geriatr Cardiol. 2014;1 1(4):316-28. doi:10.11909/j.issn.1671-5411.2014.04.007.

22. Davis KK, Himmelfarb CR, Szanton SL, Hayat MJ, Allen JK. Predictors of heart failure self-care in patients who screened positive for mild cognitive impairment. J Cardiovasc Nurs. 2015;30(2):152-60. doi:10.1097/ JCN.0000000000000130.

23. Reis HJ, de Oliveira AC, Mukhamedyarov MA, Zefirov AL, Rizvanov AA, Yalvaç ME, et al. Human cognitive and neuro-psychiatric bio-markers 
in the cardiac peri-operative patient. Curr Mol Med. 2014;14(9):1155-63. doi:10.2174/1566524014666140603114655.

24. Kramberger MG, Kåreholt I, Andersson T, Winblad B, Eriksdotter M, Jelic V. Association between EEG abnormalities and CSF biomarkers in a memory clinic cohort. Dement Geriatr Cogn Disord. 2013;36(56):319-28. doi:10.1159/000351677.

25. Cai Z, Wang C, He W, Tu H, Tang Z, Xiao M, et al. Cerebral small vessel disease and Alzheimer's disease. Clin Interv Aging. 2015;10:1695-704. doi:10.2147/CIA.S90871.

26. Schmidt MT, Kanda PA, Basile LF, da Silva Lopes HF, Baratho R, et al. Index of alpha/theta ratio of the electroencephalogram: a new marker for Alzheimer's disease. Front Aging Neurosci. 2013;5:60. doi:10.3389/ fnagi.2013.00060.

27. Aoki Y, Kazui H, Pascal-Marqui RD, Ishii R, Yoshiyama K, Kanemoto $\mathrm{H}$, et al. EEG resting-state networks in dementia with lewy bodies associated with clinical symptoms. Neuropsychobiology. 2019;77(4):20618. doi:10.1159/000495620.
28. Tarasova IV, Trubnikova OA, Barbarash OL. EEG and clinical factors associated with mild cognitive impairment in coronary artery disease patients. Dement Geriatr Cogn Disord. 2018;46(5-6):275-84. doi:10.1159/000493787.

29. Moretti DV, Pievani M, Pini L, Guerra UP, Paghera B, Frisoni GB. Cerebral PET glucose hypometabolism in subjects with mild cognitive impairment and higher EEG high-alpha/low-alpha frequency power ratio. Neurobiol Aging. 2017;58:213-24. doi:10.1016/j.neurobiolaging.2017.06.009.

30. van den Berg E, Geerlings MI, Biessels GJ, Nederkoorn PJ, Kloppenborg RP. White matter hyperintensities and cognition in mild cognitive impairment and Alzheimer's disease: a domain-specific meta-analysis. J Alzheimers Dis. 2018;63(2):515-27. doi:10.3233/JAD-170573.

31. Sheorajpanday RV, Mariën P, Nagels G, Weeren AJ, Saerens J, van Putten MJ, et al. Subcortical vascular cognitive impairment, no dementia: EEG global power independently predicts vascular impairment and brain symmetry index reflects severity of cognitive decline. J Clin Neurophysiol. 2014;31(5):422-8. doi:10.1097/WNP.0000000000000060. 